\title{
Optical Force-Induced Dynamics of Assembling, Rearrangement, and Three-Dimensional Pistol-like Ejection of Microparticles at Solution Surface
}

Jia-Syun LU, ${ }^{1}$ Tetsuhiro KUDO, ${ }^{* 1}$ Boris LOUIS, ${ }^{2,3}$ Roger BRESOLÍ-OBACH, ${ }^{* 1,2}$ Ivan G. SCHEBLYKIN $^{3}$, Johan HOFKENS, ${ }^{* 2,4}$ and Hiroshi MASUHARA*1,5

${ }^{1}$ Department of Applied Chemistry, College of Science, National Chiao Tung University, Hsinchu 30010, Taiwan

2 Department of Chemistry, Katholieke Universiteit Leuven, Heverlee B-3001, Belgium

${ }^{3}$ Division of Chemical Physics and NanoLund, PO Box 124, Lund University, 22100, Sweden, ${ }^{4}$ Max-Planck-Institute for Polymer Research, Mainz 55128, Germany

${ }^{5}$ Center for Emergent Functional Matter Science, National Chiao Tung University, Hsinchu 30010, Taiwan

\section{Corresponding Authors}

*E-mail: masuhara@masuahra.jp, kudo@toyota-ti.ac.jp, roger.bresoliobach@,kuleuven.be, johan.hofkens@kuleuven.be 


\begin{abstract}
Optical trapping and assembling dynamics of polystyrene (PS) microparticles (MPs) of $1 \mu \mathrm{m}$ diameter is studied at its solution-air surface using a widefield microscope. Upon switching on the intense $1064 \mathrm{~nm}$ laser, the MPs are gathered, forming a single concentric circle (CC)-like assembly larger than the focus. It consists of a few tens of MPs and the central part of the assembly shows structural color, which indicates that the assembly is also growing in the axial direction.-The MPs are dynamically fluctuating in inside the assembly, and some of them are ejected when newly coming MPs collide with the CC-like assembly from the bulk solution. The MPs speedily leaving the assembly are aligned in a linear manner, which we refer to as "pistole-like ejection". The three-dimensional (3D) dynamics was elucidated by changing laser power, MP concentration, and surface chemical property. It is directly observed that the trapping laser was scattered radially from the CC-like assembly and the ejection was induced along the scattered laser path. This pistol-like ejection is stochastically repeated upon the collision. After prolonged irradiation, the assembly rearranges to a hexagonal close packing (HCP)-like assembly, in which no pistol-like ejection was observed. We note that our observation is characteristic of the solution surface and were never observed in bulk solution. We conclude that the kinetically driven assembly formation gives rise to a CC-like structure which is meta-stable and shows the pistol-like ejection phenomenon. Later, the assembly rearranges to a thermodynamically stable HCP-like assembly. The assembling, pistol-like ejection, and its rearrangement are all driven by optical force, which is common for optical trapping-induced molecular crystallization and optically evolved assembling and swarming of gold nanoparticles (NPs).
\end{abstract}

\title{
1. Introduction
}

When an intense laser beam is focused on small microscale objects, optical force is exerted on them, enabling their individual manipulation. This was first proposed and demonstrated for polymer microparticles (MPs) by Ashkin and coworkers in $1986^{1}$. This phenomenon, now referred to as optical tweezers or optical trapping, and was applied to many research fields, particularly to biophysics and biochemistry ${ }^{2-9}$. Single living cells, viruses, and bacteria ${ }^{10,11}$ are historically representative examples for optical manipulation. One of the authors (H.M.) introduced a Galvano mirror set to microscope systems and achieved free manipulation of single MPs and singlet liquid droplets in the focal plane ${ }^{12,13}$. It was integrated with fluorescence spectroscopy ${ }^{14}$, time-resolved absorption spectroscopy ${ }^{15}$, microelectrochemistry ${ }^{16}$, laser ablation ${ }^{17}$, and photopolymerization ${ }^{18}$. Then, various chemical 
phenomena were explored and analyzed by utilizing optical forces. Our results of single microparticles and single microdroplets based on optical trapping were summarized ${ }^{19,20}$ and introduced as microchemistry by Ashkin ${ }^{21,22}$.

The optical forces exerted on MPs consists of gradient and scattering forces. Optical trapping is made possible when the former gradient force is larger compared to the scattering force tatter. This is typically the case in bulk solution, however, the conditions are modified at airliquid $^{23,24}$, liquid-liquid ${ }^{25}$, and solid-liquid ${ }^{26,27}$ interfaces, where the scattering force toward the interface can also immobilize the object. Thus, even small objects can be trapped at the interface with a relatively weak gradient force, which is not possible inside bulk solution and leads to new optical assembling phenomena ${ }^{24,25}$. Recently plasmonic trapping has received much attention because small objects can be easily trapped and manipulated. Conventionally metal nanostructures are prepared on glass substrates ${ }^{28-32}$ and semiconductor needles are fabricated $^{33,34}$, so that the interface effects may contribute to their trapping phenomena in addition to enhanced optical trapping force. To elucidate the role of the solution surface in optical trapping, we studied polystyrene (PS) NPs with the diameter of $200 \mathrm{~nm}$ which forms a layer-by-layer structure at solution/air interface expanding laterally over $10 \mu \mathrm{m}^{35}$. For PS NPs with the diameter of $500 \mathrm{~nm}$, a periodically arranged assembly is prepared at the solution-glass interface. The assembly displays horn-like arrays of linearly aligned NPs extending from the edge of the assembly ${ }^{26}$. The initially trapped NPs at the interface scatter the trapping laser to the outside, the scattered laser propagates through the assembled NPs and traps newly coming NPs even outside the focus. Finally, a disk-like shape with a diameter larger than $10 \mu \mathrm{m}$ is obtained.

In the case of gold (Au) NPs, we reported that the NPs are initially aligned perpendicular to the laser polarization inside the irradiated area due to optical binding. The formed periodical structure scatters the trapping laser strongly. Consequently many Au NPs are collected around the structure and fluctuate vigorously like a group of bees ${ }^{36}$. We call this phenomenon "optically evolved assembling and swarming" of Au NPs and demonstrate that its morphology depends on trapping laser polarization and power. The scattering efficiency is high particularly when the trapping laser wavelength matches with surface plasmon resonance band, leading to more active swarming ${ }^{37}$. The swarming dynamics and mechanism are studied systematically, for example, their dynamic coupling with local phase separation in polymer solution was elucidated, confirming that photothermal heating of the trapped Au NPs 
is also involved ${ }^{38}$.

This optical scattering and propagation at interface holds also for molecular solution, which explains the molecular crystallization of amino acids ${ }^{23,39}$ and inorganic compounds such $\mathrm{KCl}^{40}$. We have demonstrated that the optically generated crystals are much larger than the focused laser beam size (about $1 \mu \mathrm{m}^{2}$ ) and they can even enlarge up to a few tens of $\mu \mathrm{m}^{2}$. It is worth noting that this crystallization was observed not only under super-saturated condition but also in unsaturated solution. Like the previous cases of PS NPs ${ }^{26}$, the scattered trapping laser from the focus propagates through the prepared crystal and forms an attractive potential at its surrounding edge. Namely the optical potential leaks out from the focal volume to the edge of the crystal, so that the crystal growth outside the focal volume is made possible even for unsaturated solution conditions ${ }^{39}$.

Therefore, the assembling of MPs and NPs as well as molecular crystallization by optical force should have the same origin and studying on their dynamics and mechanism is important to understand the phenomena from a chemical viewpoint. Here, we study how small objects are assembled and rearranged at solution surface by using $1 \mu \mathrm{m}$ PS MPs. They are visible and easy to follow one-by-one in two-dimensional and 3D images. We have succeeded to show that after switching on the trapping laser, the initially formed assembly has a concentric circular (CC)-like structure and it stochastically rearranges to a similar CClike or hexagonal close packed (HCP) structure. The rearrangement is coupled with a pistollike ejection of some MPs, and everything is driven by optical forces. The ejection process is characteristic of the solution-air surface and thus never happens at the solution-glass interface nor inside bulk solution. The pistol-like ejections are intrinsically 3D and their dynamics is elucidated by a multiplane widefield microscopy ${ }^{41}$ utilizing fluorescent PS MPs.

\section{Experimental}

\subsection{Materials and sample preparation}

All the PS MPs used in this work are commercially available. The main PS MPs used in this work are $1 \mu \mathrm{m}$ non-fluorescent PS MP (Polysciences, \#07310-15, sulfate ester). Additionally, $1 \mu \mathrm{m}$ non-fluorescent PS MP-COOH (Polysciences, \#08226-15, carboxylated) was used for examining the surface charge effect, and $1 \mu \mathrm{m}$ fluorescent PS MP (Yellow-green fluorescent (505/515), ThermoFischer Scientific, FluoSpheresTM; F8823) was used for 3D fluorescence analysis. We diluted the commercial samples with neat $\mathrm{D}_{2} \mathrm{O}$ to a final MPs concentration in the 
range from $1.1 \times 10^{-4}$ to $2.8 \times 10^{-5} \mathrm{MPs} / \mu \mathrm{m}^{3}$. This means that no PS MP is distributed at the focus before laser trapping, as the focal volume is in the order of $\mu \mathrm{m}^{3}$. We used $\mathrm{D}_{2} \mathrm{O}$ instead of conventional $\mathrm{H}_{2} \mathrm{O}$ to avoid the optical heating by the $1064 \mathrm{~nm}$ trapping laser. It is reported that a vibrational overtone of $\mathrm{OH}$ stretching mode of is situated at $1064 \mathrm{~nm}$, leading to temperature elevation of $23^{\circ} \mathrm{K}$ per $1 \mathrm{~W}(1064 \mathrm{~nm} \text { laser power })^{42}$. The $1 \mu \mathrm{m}$ PS MPs are homogeneously dispersed in the solution after the preparation due to the small density difference of PS (1.05 $\mathrm{g} / \mathrm{ml})$ and $\mathrm{D}_{2} \mathrm{O}(1.11 \mathrm{~g} / \mathrm{ml})$. The sample chambers used in this work were hand-made. First, we cleaned the glass coverslips by immersing them in 5\% detergent solution (Hellmanex III, Hellma) for overnight. Later, we glued the bottom coverslip with a glass ring (diameter $3.5 \mathrm{~cm}$; thickness $1.5 \mathrm{~cm}$ ) which carries out the function of spacer. Finally, the PS MPs solution was sonicated for $15 \mathrm{~min}$, and immediately after that, $20 \mu \mathrm{L}$ of the MPs suspension was poured inside the glass ring and covered with another coverslip to avoid $\mathrm{D}_{2} \mathrm{O}$ evaporation (Figure 1).

\subsection{Optical setup for laser trapping}

Briefly, a $1064 \mathrm{~nm}$ continuous wave laser was focused at the solution surface (upper $\mathrm{D}_{2} \mathrm{O}$ air interface) through an air-immersion objective lens (60x; NA 0.90; Olympus UPlanFLN 60X). The trapping laser power was controlled by using a half wavelength plate combined with a polarizer. Both linearly and circularly polarized laser were used as the trapping light source, and similar results are obtained.

The 2D observation was performed using an inverted widefield/transmission microscope (Figure 1a) in National Chiao Tung University.-The experimental room temperature was set to $25 \pm 1^{\circ} \mathrm{C}$. For transmission image acquisition, the sample chamber was illuminated with a halogen lamp from the top. The transmitted light was collected by the air-immersion objective, filtered with a $1010 \mathrm{~nm}$ short-pass optical filter (FF01-1010/SP-25, Semrock) to remove the backscattered light from the $1064 \mathrm{~nm}$ trapping laser, and recorded using a charge-coupled device (CCD) camera (WAT-231S2, Watec France; $30 \mathrm{fps}$ ). For recording the $1064 \mathrm{~nm}$ backscattered light from the generated assembly, we removed the $1010 \mathrm{~nm}$ short pass filter and replaced the CCD camera for a near-infrared high-sensitive camera (WAT-902H2 ULTIMATE, Watec France; 30 fps).

The 3D fluorescence imaging was performed using a home-built wide-field setup with multiple plane acquisition ( 500 pixels $\times 500$ pixels x 8 planes; 50 × 50 × $3 \mu \mathrm{m}$ ) in Katholieke Universiteit Leuven, based on a system previously described ${ }^{41}$ (Figure 1b). Briefly, a $488 \mathrm{~nm}$ laser line (100 
$\mathrm{mW}$, SpectraPhysics) was used as widefield laser illumination by focusing it at the back focal plane of the objective lens. Excitation laser power was controlled using neutral density filters. The fluorescence emission was collected by the objective and filtered with a band pass filter (ZET488/561m, Chroma Technology) and $1010 \mathrm{~nm}$ short-pass optical filter (FF01-1010/SP25 , Semrock). The emitted light goes through a set of lenses in telecentric $4 \mathrm{f}$ configuration [Objective - Lens1 - xy-Slit - Lens2 - Tube lens - Camera]. Between the last lens and the cameras, we placed a proprietary prism, which splits the entering photon beam to eight different beams with slightly different optical path length. The different imaging planes are recorded by 2 scientific Complementary metal-oxide-semiconductor (sCMOS) cameras (4 imaging planes for each camera; Orca Flash 4.0, Hamamatsu Photonics Inc.; 100 fps). The image processing and the tracking of the fluorescent particles was performed as previously reported ${ }^{41}$. Finally, we filtered the pistol-like ejected trajectories from all the obtained traces by considering the spatial-temporal characteristics of each trace.

\section{Results}

\section{3-1 Assembling, Pistol-like Ejection, and Rearrangement of MPs}

We focus an intense $1064 \mathrm{~nm}$ laser of $1.4 \mathrm{~W}$ power at the solution/air interface, which corresponds to the power density of about $140 \mathrm{MW} / \mathrm{cm}^{2}$. The $1 \mu \mathrm{m}$ PS MPs are gathered individually from the bulk solution, leading to the formation of a single large assembly. The laser power is much higher than the minimum required power needed to immobilize a single 1 $\mu \mathrm{m}$ PS MP at the interface. The assembly evolved with irradiation time, showing an increase in lateral size and rearrangements of the packing structure of the PS MPs. A few PS MPs are sometimes ejected in a linearly aligned manner from the assembly to the outside, and the ejection directionality and speed is high and fast, respectively. It is worth noting that the phenomenon is stochastically reproducible. Their dynamical behavior of PS MPs changes from measurement to measurement due to stochastic origin, whose representative sequential transmission images are shown in Figure 2. Upon starting the irradiation of the sample with the trapping laser, several PS MPs were captured rapidly at the focal spot. The size of the assembly became larger than the focus in a few seconds and close to $10 \mu \mathrm{m}$ at $10 \mathrm{~s}$, giving a CC-like packing (Figure $2 \mathrm{C}$ ). These results are obtained with a linearly polarized trapping laser, and a similar behavior was also observed using a circularly polarized laser. However, circularly polarized trapping laser also induces a rotation of the CC-like assembly. This makes images less clear and the analysis more difficult. Therefore, all the data shown in this work were obtained with a linear polarization. We followed the assembly growth process "by eye" frame 
by frame and counted how many MPs join the assembly (Figure S1). After $30 \mathrm{~s}$, the single assembly consists of a few tens of MPs or more. The outside border of the assembly looked clear, while its central part relatively dark and vague, which indicates that the MPs at and around the focus are three-dimensionally aggregated and partly well packed, forming a single assembly. Sequentially, few MPs are ejected out radially from the assembly in a linear alignment (Figure 2D), where these ejected MPs moved as a group. When the MPs arrived far from the focus, its linear morphology disappeared, separating to individual MPs due to viscous drag of the solvent. During the ejection, the MPs are always linearly aligned, where the first MP moved at the high speed and several MPs followed its direction. We refer this new phenomenon as "pistole-like ejection" and we consider that the high directionality and linear morphology are favored by the hydrodynamic effects, since it refer less resistance from the solution compared to other aggregation morphology of the ejected PS MPs.

After long trapping laser irradiation, the CC-like assembly is turned into a hexagonal closepacking (HCP) structure (Figure 2F). Most PS MPs appeared clear in HCP-like assembly as opposed to the CC-like assembly, indicating that they were mainly well arranged as a single layer at the solution surface. It is worth noting in Figure $2 \mathrm{G}$ that the pistol-like ejection events (e.g. from $\mathrm{C}$ to $\mathrm{D}$ ) behavior happened only when the CC-like assembly was larger than a certain size (about $55 \mu \mathrm{m}^{2}$ ). Once the assembly reaches this minimum size, it becomes unstable and the MPs begin to dynamically fluctuate. Structural rearrangement from the CC-like to the HCP structures is always coupled to a pistol-like ejection of several PS MPs. By checking the images frame by frame (the frame rate was $30 \mathrm{fps}$ ), one pistol-like ejection was observed within 0.5 sec or shorter after the rearrangement from the CC to HCP structures (SI Video1). Once we doubted if a CC-like optical interference pattern of the trapping laser is generated at the solution surface $^{43}$ and MPs are trapped there, leading to the formation of the CC-like assembly. The possible interference pattern should not be modified, as far as optical condition is not changed. Here, the PS MPs often rearranged from one CC-like to HCP or other CC-like structures. Thus, the optical interference is not a reason for the present CC-like assembly formation.

We consider that the CC-like structure is kinetically formed, namely, MPs are attracted to the focus by laser irradiation and sequentially attached to the aggregated MPs. A certain number of PS MPs are located below the surface, and some of them may be ejected upon the rearrangement to the 2D HCP structure. This happened around $70 \mathrm{sec}$ for the present case, and the size of the HCP assembly gradually increased with the irradiation time. The formed HCP 
assembly grew with further irradiation, but did not usually yield the pistol-like ejection. This can be quite reasonable, as it is well known that 2D HCP structure of PS MPs is stable and thermodynamically favored at interface ${ }^{44,45}$. The present behavior obtained with continuous irradiation is summarized in Figure 2G.

Even if HCP did not yield pistol-like ejections, they could be rarely observed for the HCP structure when we turned off the trapping laser and on it again with a $5 \mathrm{~s}$ interval (Figure S3). The optically induced assembly was quickly formed again, and it always showed at least partly the HCP structure. Upon switching off the laser, random diffusional dispersion of the PS MPs is observed, but switching it on again pushed the MPs back to form an assembly again. The local concentration of the MPs around the focus is high, so the assembling process is now thermodynamically controlled. This dispersion from the HCP structure and reassembling to the $\mathrm{HCP}$ did not reproduce the exact same structure, which may be ascribed to the $3 \mathrm{D}$ diffusional motion after the switching off the irradiation.

\section{3-2 Origin of the Pistol-like Ejection Behavior}

The pistol-like ejection is always observed radially from the center to the outside, which can be ascribed to the scattering and its propagation of the trapping laser inside the CC assembly. Occasionally we found that, before the $1 \mu \mathrm{m}$ PS MPs are ejected out, they form a horn-like alignment. This is similar to what was observed for $200 \mathrm{~nm}$ PS NPs at solution surface ${ }^{35}$ and $500 \mathrm{~nm}$ PS NPs at solution-glass interface ${ }^{26}$. Therefore, the pistol-like ejection could also be due to the scattering and propagation of the laser. To confirm this idea, we observed not only transmission but also backscattering images under similar condition. Figure 3 and SI Video2 show a representative example provided by overlaying the backscattering images of $1064 \mathrm{~nm}$ trapping laser on the conventional transmission images. We observed that the laser scattering was always expanded in multiple radial white streams from the center of the CC-like structure. However, its scattering direction rotated together with the rearrangement of the structure. One can also see in Figure 3 that the MPs are ejected similarly in a radial manner as the $1064 \mathrm{~nm}$ light propagates. Based on the observed results, we propose that the scattered light of the trapping laser from the central part of the CC-like assembly and its directional propagation through the structure are the origin of the pistol-like ejection at solution surface.

\section{3-3 Two-dimensional Analysis of Pistol-like Ejection Behavior}

The CC-like assembly does not always undergo the rearrangement to HCP structure but 
remains with a little bit modified CC-like arrangement even through the pistol-like ejection. In this case the single CC-like assembly could repeat the pistol-like ejection. It is important to point out that, although many PS MPs are trapped, the single assembly formation is a stochastic phenomenon. In Figure 4 shows two representative examples of an assembly rearrangement process, yielding the pistol-like ejection phenomenon. Four ejection events were simultaneously observed from Figure $4 \mathrm{~A}$ to $4 \mathrm{~B}$ with the total number of 18 ejected MPs. A single ejection event was observed from Figure 4C to 4D with 8 MPs ejected. The ejection was induced with high directionality, showing a straight line radially expanding from the assembly to the outside (Figure S2 and Video 2). We counted the number of the ejection events observed in each frame and the number of the PS MPs per ejection event, and the result is summarized in a $3 \mathrm{D}$ graph in Figure 4E. Both numbers changed from measurement to measurement, because of the stochastic nature of the single assembly formation and rearrangement. Here a few tens of events were induced during 3 min, more than half of the events ejected a few MPs, and the ejection of a single MP was observed frequently. The results on four ejection events in one video frame giving 8 MPs (Figure 4A to 4B) and single ejection with 8 MPs (Figure 4C to 4D) are marked in Figure 4E. Their pistol-like ejection is clearly demonstrated to be very efficient.

To understand the pistol-like ejection better, we analyzed the dynamics as a function of the laser power as given in Figure 5. Generally, the ejection frequency is greatly reduced when the trapping laser power is decreased from $1.4 \mathrm{~W}$ to $0.1 \mathrm{~W}$. It is worth noting that the ejection events of a single MP were only observed at $1.4 \mathrm{~W}$, while the events with a few MPs were observed at powers of $0.6 \mathrm{~W}$ and $0.1 \mathrm{~W}$. In case of high power, the scattering light intensity is also high, so even a single PS MP can receive enough force to be ejected. We consider that, as the scattering force is increased for the larger target, a larger volume containing a few MPs is necessary for receiving strong enough optical force to observe pistol-like ejection at the low laser power. This power dependence also supports our mechanism that the pistol-like ejection is directly induced by the scattering of the trapping laser.

We analyzed the ejection velocity and the motion by tracking the $2 \mathrm{D}$ ejection motion using the software ImageJ. Due to the 3D morphology of the CC-like assembly, the ejected PS MPs were not always located on the focal plane, but in this section (see the next section for a detailed 3D analysis) we tracked only laterally the first MP of each pistol-like ejection event and estimated its velocity. The moved distance for the first MP was measured from 
every two frames and the speed was calculated by dividing the tracked motion with the frame time. We analyzed the initial 7 to 9 frames in which almost all the pistol-like ejection trajectory is completed. The fastest velocity was obtained at the beginning of ejection behavior and then the speed dramatically slowed down with time and distance as shown in Figure 5E. We consider that the ejection behavior is completed when the linear morphology disappeared giving individual MPs in Brownian motion. This was observed around 500 $800 \mathrm{~ms}$ (15-24 frames) after the ejection was started. The 2D velocity of the first MP of the longer alignment (more MPs) is faster than that of a shorter one (less MPs). This is probably related to how the scattering force is exerted on the ejected MPs, that is, stronger force is exerted on a larger aggregate.

To support the present explanation for Figure 5, we studied the dynamics by tuning MP concentration. The results are summarized in Figure S4. As mentioned earlier, the incorporation of MPs into the CC-like assembly triggers the pistol-like ejection, so that the ejection phenomena can be modified by changing the MP concentration. We used three different MPs concentrations while maintaining the laser power at $1.4 \mathrm{~W}$ (Figure S4), which was the same power used in the above described results. The higher MPs density sample shows more often the ejection events and gives more ejected MPs for each ejection. A high concentration will obviously lead to more speedy trapping of MPs, and thus more MPs will be gathered at the focus and will more often collide to the bottom of the assembly. Therefore, the more frequent ejection event in the high concentrated solution is likely due to the higher probability of collision between the assembly and newly coming MPs. For lower concentration the ejection is still observed, while it happened not with a single MP. The collision of a single MP to the assembly does not give enough momentum, while the assembling growth proceeds relatively slow in individual trapping manner. Thus a few MPs must receive a larger momentum for triggering the rearrangement.

In general, an assembly of colloidal PS MPs is formed and stabilized based on the balance between their attractive and repulsive forces due to their surface charge and van der Waals force, respectively. Thus, it is interesting to compare the present pistol-like ejection dynamics with that of PS MPs having different surface properties. Here, we introduced a $1 \mu \mathrm{m}$ PS MPs whose surface is modified with carboxylate moiety (referred to as PS MP-COOHs) and compared its behavior with that of the PS MPs studied. The $\zeta$-potential of PS NP-COOH and the PS MPs is 
$-40 \mathrm{mV}$ and $-33 \mathrm{mV}$, respectively. Altough both MPs gave the similar CC-like assembly, their apparent stability was different from one another. The size change of the PS MPs assembly is relatively large compared to PS PM-COOHs. The PS MPs showed less frequent ejection with more ejected PS MPs per the ejction event, while the PS PM-COOHs showed a larger number of ejection events but with a lot of single particle ejections (Figure S4). It is considered that PS MP-COOHs are relatively more repulsive (i.e. larger $\zeta$-potential) and thus less aggregated inside the a single assembly. Thus, the ejection of PS MP-COOHs consists of smaller particle numbers and subsequently their assembly size flucuated less frequently.

\section{3-4 Three-dimensional Dynamics of Pistol-like Ejection Behavior}

From the change in MPs focal position during the ejection event (Figures 4B and 4D) we can easily deduce that the pistol-like ejection is intrinsically a 3D phenomenon. Indeed, the expelled MPs were defocused after the ejection event, which implies that the MPs were thrown out with some small angle with respect to the solution surface. Therefore, we studied the 3D nature of the ejection behavior by using our recently developed widefield multiplane microscope (Figure 1: Detection Path b) $)^{41}$. Because our 3D in-house tracking algorithm can only be used with fluorescent MPs, we prepared a 1:9 mixture of fluorescent and nonfluorescent $1 \mu \mathrm{m}$ MPs keeping the total number of the PS MPs constant. The idea is that we generate the same assembly as previously described but only looked at the fluorescent MPs trajectories. while the laser power was decreased to $0.36 \mathrm{~W}$ to reduce the number of ejection events per time unit, which makes accurate analysis easier. Under these conditions the CClike assembly formation and the pistol-like ejection were well reproduced and analyzed. The 3D microscopy setup consists of two cameras which can observe the same volume; one captures transmission images while the other does fluorescence images. In Figure S5, both images showing that one MP is ejected from the edge of the CC-like assembly are overlaid. Using this type of acquisition, it is possible to gain insight into the positions from where particles are ejected from the assembly and we found that almost the MPs are ejected from the edge of CC-like assembly. In addition, we will be able to determine the out-of-plane angle of the pistol-like ejection and characterize the full 3D profile of the ejection.

To characterize the 3D motion of the ejection, we repeated the experiment using only $1 \mu \mathrm{m}$ fluorescent PS MPs and we changed the camera configuration to be able to register a full $3 \mathrm{D}$ image $(50 \times 50 \times 3 \mu \mathrm{m})$. Fluorescence images of the CC-like assembly before and after 
the ejection at two different focal planes (z-position) are given in Figure 6. The trapping laser is focused on solution surface (first imaging plane; $\mathrm{z}=0 \mu \mathrm{m}$ ) and each PS MP is clearly observed before ejection. As a visual aid, the ejected MPs located at the edge of the assembly are surrounded by red circles. Before the ejection, these "expelled" MPs were well focused at the first imaging plane $(\mathrm{z}=0 \mu \mathrm{m})$, while after they moved to the right side and out of focus (Figure 6A-B). Instead, if we simultaneously follow the same event at the last imaging plane $(\mathrm{z}=-2.8 \mu \mathrm{m})$, we observed that most PS MPs are not well focused, as they are located at the surface and the PS MPs in red circles looked vague before the ejection but became clear (i.e. focused) after the ejection (Figure 6C-D). Thus, we understand that the PS MPs are ejected three-dimensionally from the solution surface to the right side declining lower up to a depth of approximately $-3 \mu \mathrm{m}$.

The results obtained using multiplane microscopy are consistent with those obtained by conventional 2D imaging. However in this set of experiments, more information is contained in the image because we can extract the 3D position of every MP at each frame (as a function of time), so that we are able to reconstruct how the trapped PS MPs are ejected three dimensionally and estimate their speeds. We tracked the motion of all the detected fluorescent MPs and obtained the traces of the ejected MPs by filtering them according to their characteristic motion. The 3D pistol-like ejection behavior of individual fluorescent MPs is shown in Figure 6E, where the depth position is given by color-coding on the $2 \mathrm{D} x$ y plane. Just below it, the projection of the same data on the radial-z plane is also represented. The MPs were initially located around the focus at the solution surface and radially descended to $2-3 \mu \mathrm{m}$ below the surface. As the ejection took place in all the directions, we replotted the trajectory simply as functions of the depth and the radial distance from the focal center. As shown in Figure 6F, the MPs initially showed similar descending but were dispersed after traveling $4 \sim 5 \mu \mathrm{m}$ from the edge of the assembly. The MPs are ejected by the radially pushing scattering force of the trapping laser and their speed slowed down due to the solvent resistance, losing its clear linear directionality. It appears that the MP moved upward after the travelling of $4 \sim 5 \mu \mathrm{m}$, which could be ascribed to the density difference between lighter PS MP and heavier $\mathrm{D}_{2} \mathrm{O}$. From this idea we could determine that the MPs are ejected in a straight-line trajectory with an angle of $11^{\circ} \sim 15^{\circ}$ with respect to the $\mathrm{D}_{2} \mathrm{O}$ /air interface and this angle does not depend on the trapping laser power $(0.36,0.60$ and $0.84 \mathrm{~W})$, which suggests that non-optical forces and/or the intrinsic geometry of the CC-like structure might play an 
important role on the direction of the ejection. The ejection speed was also obtained as a function of time (Figure $6 \mathrm{G}$ ), showing a similar trend as the result obtained in the 2D analysis.

\section{Discussion}

We have described the optical trapping-induced assembling and rearrangement of $1 \mu \mathrm{m}$ PS MPs at $\mathrm{D}_{2} \mathrm{O}$ solution surface. A single CC-like assembly was initially formed from the incoming MPs attracted by the trapping laser. Inside of the assembly, the MPs fluctuate and yield the pistol-like ejection in a radial directionality from the assembly to the outside. The number of PS MPs per ejection event as well as the number of the ejection events observed in each frame were analyzed as a function of the irradiation time. Sequentially, the MPs were ejected in a completely straight linear manner, then gradually losing their speed, reaching a distance of a few tens $\mu \mathrm{m}$, before dispersing to individual MPs displaying Brownian motion. Upon the pistol-like ejection the CC-like assembly rearranged to either other CC-like assembly or to more thermodynamically favored HCP-like structure. These dynamic behaviors are summarized and illustrated in Figure 7. The ejection speed for the highest laser power $(1.4 \mathrm{~W})$ was estimated to be $200 \mu \mathrm{m} / \mathrm{s}$, which is much faster compared to the nondirectional state-Brownian diffusion. The assembling and rearrangement phenomenon of MPs is characteristic of optical trapping at solution surface. However, the pistol-like ejection itself is intrinsically 3D. This unique phenomenon is understandable using the knowledge from as optically evolved assembling. Indeed, in a similar way, the pistol-like ejection is driven by the propagation of the scattered $1064 \mathrm{~nm}$ trapping laser through the assembly. In competition with the pistol-like ejection, the CC-like assembly continues to grow due to the incorporation of newly attracted MPs. When the assembly reaches a certain size, it rearranges to an HCPlike structure which is accompanied with an additional pistol-like ejection. We consider that the rearrangement takes place from the kinetically controlled assembly (CC-like) to the thermodynamically stable one (HCP-like).

Our understanding of the rearrangements and pistol-like ejection is schematically illustrated in Figure 7. After the initial stage (Figure 7A-7B) in which the assembly reaches a certain size, the pistol-like ejection starts to be observed. It is important to note that the pistol-like ejection of MPs was observed upon the incorporation of new MPs to the assembly. Some dark MPs rapidly approached to the assembly from bulk solution and crashed into the bottom of the assembly. Immediately after, the CC-like assembly started to rearrange back to another 
CC-like configuration (Figure 7 (C) -> (B)) or less probably to HCP structure if its size is big enough (Figure 7 (C) -> (D)). The first step is the destabilization of the assembly by new incorporation MPs which are attracted by the optical force (shown in SI Video1). Then, these MPs impact with the assembly, and their kinetic momentum is transferred to the assembly, leading to its destabilization. The second step is the ejection of some MPs from the assembly to release their excess energy. Some MPs cannot find a stable position inside the CC-like assembly and are consequently kicked out of the assembly by the scattered light propagation of the trapping laser. The ejections always occurred with high linear directionality, following the scattering of the laser and gradually undergoing dispersion. The third step is the rearrangement of the assembly. As the ejection of few PS MPs may generate some holes in the assembly structure, the newly joining MPs can find a stable position within the assembly, starting the rearrangement process and leading to a more stable and larger structure with $\mathrm{CC}$ like or HCP like assembly ((Figure 7 (C) -> (B) or (C) -> (D)). It is worth noting that most of the MPs inside the assembly were blurred in the transmission images, which means that the MPs motion is faster than the acquisition time of the camera (33 ms), confirming the expected vigorous movement during the rearrangement.

Information on the 3D aspect of the pistol-like ejection was obtained using our recently developed widefield multiplane microscope. It is surprising that the ejection takes place with a very sharp angle of $11^{\circ} \sim 15^{\circ}$ with respect to the solution surface. The NA value of the $60 \mathrm{x}$ airimmersion objective lens used in this work is 0.90 , so that the Gaussian beam of the trapping laser illuminates within the range of $45^{\circ} \sim 135^{\circ}$ relative to the surface. Thus, the pistol-like ejection occurs only within a small angle outside the irradiated trapping laser cone. When the ejection starts, a single disc-like CC-like assembly of the MPs has already formed eonstructed and may be mechanically rigid. With a very sharp angle (less than $11^{\circ}$ ), the ejected PS MPs would receive an additional frictional resistance from the assembly. Therefore, the ejection would become easier for larger angle but it did not occur at angle larger than $15^{\circ}$. This may be ascribed to different reasons. One possibility is based on our former experiment of gold NPs inside solution ${ }^{46}$. The trapping laser was focused sharply, and the distribution of gold NPs was examined by shifting the focal plane. No gold NP was found not only near the focus but also at the irradiated areas much lower than the focal point, whose depth reached to a few tens of micrometers. Namely, the scattering force pushed all the gold NPs to the focus in a large conelike space. Similarly, the PS MPs are pushed up inside the cone-like space due to gradually increasing scattering force from the bottom. This scattering force should suppress the pistol- 
like ejection toward to bottom in the cone. This can explain that the ejection does not take place with the angle range of $45^{\circ} \sim 135^{\circ}$. The directional flow of the PS NPs toward the focus would induce solvent flow inside the cone and additionally outside of the cone. This expanded flow may hamper the pistol-like ejection at the angle less than $45^{\circ}$.

At present it is not clear why no pistol-like ejection was observed for the angle range of 15 $45^{\circ}$. Here, we mention some possible reasons which will be examined by specially designed experiments. One is concerned with the optical refractive index change brought about by the assembly formation. The index of PS and water is 1.63 and 1.33, respectively, and the assembly should have the averaged value and/or show some gradient depending on the volume ratio of PS NPs and water. Optical force value and its direction change from position to position and time to time. Another reason may be related to thermal effect ${ }^{24,47,48}$, as the focused high intensity laser irradiation always results in local heating, although we use $\mathrm{D}_{2} \mathrm{O}$ to avoid the $1064 \mathrm{~nm}$ absorption by the solvent. The spatial distribution of the mechanical rigidity and refractive index of the assembly, as well as heating effects, must be dynamically balanced with each other, which is important for understanding the pistol-like ejection with a small angle distribution. The next step to reveal these dynamics is to apply the 3D multiplane microscope with different optical trapping parameters to PS MPs with different size, which is being planned in our laboratory.

In addition, we plan to introduce femtosecond laser as a trapping light source and to assemble PS MPs at solution surface. In the ensemble level we demonstrated that many PS, silica, and silane-coated silica NPs can be trapped and then ejected depending on the laser polarization. These findings are explained in terms of single transient assembly formation in the optical trap ${ }^{49-52}$. In single NPs level, Goswami et al. have reported that femtosecond laser enables manipulation and construction of $250 \mathrm{~nm}$ PS NPs with a specific 3D geometry. Not only optical trapping potential but also assembly of NPs can be controlled by femtosecond laser polarization ${ }^{53,54}$. Two-photon fluorescence is used for tracking the movement of individual NPs and for analyzing their geometry ${ }^{55}$. Even hierarchical self-assembly was demonstrated and discussed $^{56}$, which will be useful reference for fabricating the assembly as the bottom-up approach. The different optical trapping conditions are applied in the present wok and their study; at solution interface vs. in solution, by CW vs. by femtosecond laser, and for bare vs. for dye-doped particles, and in the ensemble level vs. individual particle level, but it is important how laser polarization determines respective phenomenon. We consider in the 
present system that the laser polarization loses its directionality through multiple scattering and propagation inside the disc-like CC-like assemble consisting of a few tens of MPs at the solution surface. It will be interesting and fruitful to study the trapping polarization effect on the assembly formation at solution surface individual NP level by CW and femtosecond lasers for comprehensive understanding of optical manipulation of NPs.

\section{Conclusion}

The PS MPs dynamics of their assembling and rearrangement involving the pistol-like ejection at solution surface can be triggered, maintained, assisted, supported, and/or resulted by optical force induced by a focused laser beam. The gradient force collects the incoming MPs at and around the focus. Then, the initially trapped PS MPs are assembled following a CC-like arrangement and they can further scatter the trapping laser light around the focus. Once the assembly grows through the trapping laser propagation of the trapping laser inside the assembly, some MPs aggregates in the CC-like assembly receive a strong scattering force, which can overcome the adhesion force leading to the pistol-like ejection. The ejection process reduces the energy excess of the fluctuating CC-like assembly, initiating a stabilizing rearrangement process to another CC-like or the HCP like assembly. Many ejection processes can occur until the thermodynamically stable HCP-like structure is formed as illustrated in Figure 7. This understanding is useful to explain many other optical force-induced phenomena at solution interface. For instance, optical trapping and assembling of PS MPs and NPs, optical assembling and swarming of Au NPs, and molecular crystallization could be well interpreted in terms of this optical force-induced dynamics at solution interfaces. Furthermore, the pistol-like ejection needs detailed examination as collective motion of colloidal particles where different hydrodynamic forces can be coupled with optical forces ${ }^{57,58}$ and will be a useful tool in micromachining ${ }^{59,60}$. New molecular phenomena as well as NPs and MPs dynamics are currently further explored in our group, which is opening new approaches of optical manipulation chemistry at solution surface and interface. This work is hopefully a cue in research field in optical manipulation chemistry and related technology.

\section{ASSOCIATED CONTENT \\ Supporting Information}

The Supporting Information is available free of charge on the ACS Publications website at https://..............

SI Figures and Captions for J.-S. LU et al. (PDF)

SI Videos for J.-S. LU et al. (Video) 


\section{AUTHOR INFORMATION \\ Corresponding Authors}

*E-mail: masuhara@masuahra.jp, kudo@nctu.edu.tw, roger.bresoliobach@kuleuven.be, johan.hofkens@kuleuven.be

\section{Authors}

Jia-Syun LU, Mr.

Department of Applied Chemistry, College of Science, National Chiao Tung University, Hsinchu 30010, Taiwan.

Tel: $+\underline{+886-3-5712121 \text { ext. } 56595}$

ian311.22@gmail.com

Tetsuhiro KUDO, Dr.

Laser Science Laboratory, Toyota Technological Institute,

2-12-1 Hisakata, Tempaku-ku, Nagoya 468-8511, Japan

Tel: + 886-3-5712121

kudo@toyota-ti.ac.jp

Roger BRESOLI-OBACH, Dr.

Department of Chemistry, Katholieke Universiteit Leuven,

Heverlee B-3001, Belgium

Tel: $+34-680-483-556$

roger.bresoliobach@kuleuven.be

Boris LOUIS, Mr.

Molecular Imaging and Photonics, Dept. of Chemistry, KU Leuven, Belgium

and

Division of Chemical Physics and NanoLund, PO Box 124 Lund University, 22100, Sweden boris.louis@kuleuven.be

Ivan G. SCHEBLYKIN, Prof. Dr.

Division of Chemical Physics and NanoLund, PO Box 124, Lund University, 22100, Sweden ivan.scheblykin@,chemphys.lu.se

Johan HOFKENS, Prof. Dr.

Department of Chemistry, Katholieke Universiteit Leuven,

Heverlee B-3001, Belgium

and

Max Planck Institute for Polymer Research

Tel: + 32-474-43-77-08

johan.hofkens@kuleuven.be

Hiroshi MASUHARA, Prof. Dr.

Department of Applied Chemistry, College of Science, National Chiao Tung University, Hsinchu 30010, Taiwan

and

Center for Emergent Functional Matter Science, National Chiao Tung University, Hsinchu 30010, Taiwan.

Tel: $\underline{+886-3-5712121 \text { ext. } 56593}$

masuhara@masuahra.jp 


\section{ORCID ,}

Tetsuhiro Kudo: 0000-0002-7339-7745

Roger Bresoli-Obach: 0000-0002-7819-7750

Boris Louis: 0000-0003-2882-6907

Ivan G. Scheblykin: 0000-0001-6059-4777

Johan Hofkens: 0000-0002-9101-0567

Hiroshi Masuhara: 0000-0002-4183-5835

\section{Author Contributions}

J.-S. L., T.K., R.B.-O., and B.L. conducted experiments and summarized the results. T.K., R.B.-O., and H.M. wrote the manuscript. I.G.S., J.H., and H.M. supervised the project. All authors discussed on the results and commented on the manuscript. All authors have given approval to the final version of the manuscript.

\section{Notes}

The authors declare no competing financial interest.

\section{ACKNOWLEDGMENTS}

This work is supported by Ministry of Science and Technology, Taiwan (grant No. MOST 109-2634-F-009-028) and the Center for Emergent Functional Matter Science of National Chiao Tung University from The Featured Areas Research Center Program within the framework of the Higher Education Sprout Project by the Ministry of Education (MOE) in Taiwan. Thanks are also due to funding by Ministry of Science and Technology (MOST) of Taiwan (MOST 108-2113-M-009-015- to H.M. and MOST 108-2112-M-009-008- to T.K.). J.H. gratefully acknowledges the financial support of the Flemish Government through longterm structural funding Methusalem ((CASAS2, Meth/15/04). R.B.-O. thanks the Fonds Wetenschappelijk Onderzoek-Vlaanderen for postdoctoral $(12 \mathrm{Z} 8120 \mathrm{~N})$ and long stay abroad (K1G1519N; Taiwan) fellowships.

\section{REFERENCES}

(1) Ashkin, A.; Dziedzic, J. M.; Bjorkholm, J. E.; Chu, S. Observation of a Single-Beam Gradient Force Optical Trap for Dielectric Particles. Opt. Lett. 1986, 11 (5), 288-290.

(2) Molloy, J. E.; Padgett, M. J. Lights, Action: Optical Tweezers. Contemporay Physics, 2002, 43(4), 241-258.

(3) Grier, D. G. A Revolution in Optical Manipulation, Nature 2003, 424, 810-816

(4) Dholakia, K.; Reece, P.; Gu, M. Optical micromanipulation. Chem. Rev. Soc. 2008, 37, 42-55.

(5) Moffitt, J. R.; Chemla, Y. R.; Smith, S. B.; Bustamante, C. Recent Advances in Optical Tweezers, Annu. Rev. Biochem. 2008, 77, 205-228.

(6) Dholakia, K.; Zemanek, P. Colloquium: Grip by Light: Optical Binding. Rev. Mod. Phys. 2010, 82, 1767.

(7) Fazal, F. M.; Block, S. M. Optical Tweezers study life under tension. Nature Photon. 2011, 5, 318-321.

(8) Shoji, T.: Tsuboi, Y. Plasmonic optical trapping of soft nanomaterials such as polymer chanis and DNA: Micro-patterning formation. Opt. Rev. 2015, 22, 137-142.

(9) Rodriguez-Sevvila, P.; Labrdor-Paez, L.; Jaque, D.; Haro-Gonzalez, P. Optical Trapping for Biosensing: Material and Applications. J. Mater. Chem. B 2017, 5, 9085.

(10) Ashkin, A.; Dziedzic, J. M. D.; Yamane, T. Optical Trapping and Manipulation of 
Single Cells Using. Nature 1987, 330, 769-711.

(11) Ashkin, A.; Dziedzic, J. M. D. Optical Trapping and Manipulation of Viruses and Bacteria. Science 1987, 235 (4795), 1517-1620. https://www.jstor.org/stable/1699058.

(12) Sasaki, K.; Koshioka, M.; Misawa, H.; Kitamura, N.; Masuhara, H. Laser Scanning Micromanipulation and Spatial Patterning of Fine Particles. Jpn. J. Appl. Phys. 1991, 30, L907.

(13) Sasaki, K.; Koshioka, M.; Misawa, H.; Kitamura, N.; Masuhara, H. Pattern Formation and Flow Control of Fine Particles by Laser-Scanning Micromanipulation. Optics Lett. 1991, 16, 1463.

(14) Kamada, K.; Misawa, H.; Sasaki, K.; Kitamura, N.; Masuhara, H. Picosecond Lasing Dynamics of a Single Dye-doped Microparticle in Solution. Chem. Phys. Lett., 1993, 210, 89-93

(15) Tamai, N.; Porter, C. F.; Masuhara, H. Femtosecond Transient Absorption Spectroscopy of a Single Perylene Microcrystal under a Microscope. Chem. Phys. Lett., 1993, 211, 364-370

(16) Nakatani, K.; Uchida, T.; Misawa, H.; Kitamura, N.; Masuhara, H. Laser Trapping and Electrochemistry of a Single Oil Droplet in Water: Electron Transfer across the OilDroplet/Electrode Interface. J. Electroanal. Chem. 1994, 367, 109-114.

(17) Misawa, H.; Koshioka, M.; Sasaki, K.; Kitamura, N.; Masuhara, H. Laser Trapping, Spectroscopy, and Ablation of a Single Latex Particle in Water. Chem. Lett. 1990, 1945.

(18) Ito, S.; Tanaka, Y.; Yoshikawa, H.; Ishibashi, Y.; Miyasaka, H.; Masuhara, H. Confinement of Polymerization and Solidification with Radiation Pressure. J. Am. Chem. Soc. 2011, 133, 14472-14475.

(19) Masuhara, H.; De Schryver, F. C.; Kitamura, N.; Tamai, N., Eds. Microchemistry: Spectroscopy and Chemistry in Small Domains. 1994, North-Holland, Amsterdam.

(20) Masuhara, H., De Schryver, F. C., Eds. Organic Mesoscopic Chemistry, 1999, Blackwell Science, Oxford.

(21) Ashkin, A. Optical Trapping and Manipulation of Neural Particles Using Lasers. Proc. Nat. Acad. Sci. 1997, 94 (10), 4853-4860.

(22) Ashkin, A. History of Optical Trapping and Manipulation of Smal-Neutral Particle, Atoms, and Molecules, IEEE J. Selected Topics in Quantum Electronics, 2000, 6, 841.

(23) Sugiyama, T.; Yuyama, K.; Masuhara, H. Laser Trapping Chemistry: From Polymer Assembly to Amino Acid Crystallization. Acc. Chem. Res. 2012, 45 (11), 1946-1954..

(24) Lv, C.; Varanakkottu, S. N.; Baier, T.; Hardt, S. Controlling the Trajectories of Nano/Micro Particles Using Light-actuated Marangoni Flow. Nano Lett. 2018, 18 (11), 6924-6930.

(25) Shoji, T.; Naka, T.; Koyama, S.; Kameyama, T.; Torimoto, T.; Tsuboi, Y. Optical Trapping and Semiconductor Nanoparticles at Oil-Water Interfaces with a Focused Near Infrared Laser Beam. Proc. SPIE 2018, 10712, 1071209.

(26) Kudo, T.; Wang, S.-F.; Yuyama, K.; Masuhara, H. Optical Trapping-Formed Colloidal Assembly with Horns Extended to the Outside of a Focus through Light Propagation. Nano Lett. 2016, 16 (5), 3058-3062.

(27) Königer, A.; Köhler, W. Optical Funneling and Trapping of Gold Colloids in Convergent Laser Beams. ACS Nano 2012, 6 (5), 4400-4409.

(28) Juan, M. L.; Righini, M.; Quidant, R. Plasmon Nano-optical Tweezers. Nat. Photonics 2011, 5, 349-356.

(29) Shoji, T.; K.; Saitoh, J.; Kitamura, N.; Nagasawa F.; Murakoshi, K.; Yamauchi, H.; Ito, S.; Miyasaka, H.; Ishihara, H.; Tsuboi, Y. Permanent Fixing or Reversible Trapping and Release of DNA Micropatterns on a Gold Nanostructure Using Continuous-Wave 
or Femtosecond-Pulsed Near-Infrared Laser Light. J. Am. Chem. Soc. 2013, 135, 66436648.

(30) Mototsuji, A.; Shoji, T.; Wakisaka, Y.; Murakoshi, K.; Yao, H.; Tsuboi, Y. Plasmonic Optical Trapping of Nanometer-sized J-/H-Dye Aggregates as Explored by Fluorescence Microspectroscopy, Opt. Express 2017, 25, 13617-13625.

(31) Kotsifaki, D. G.; Chormaic, S. N. Plasmonic Optical Tweezers based on Nanostructures: Fundamentals, advances and prospects. Nanophoton. 2019, 8, 12271245.

(32) Shoji, T.; Itoh, K.; Saitoh, J.; Kitamura, N.; Yoshii, T.; Murakoshi, K.; Yamada, Y.; Yokoyama, T.; Ishihara, H.; Tsuboi, Y. Plasmonic Manipulation of DNA Using a Combination of Optical and Thermophoretic Forces: Separation of Different-sized DNA from Mixture of Solution. Sci. Rep. 2020, 10, 3349.

(33) Shoji, T.; Mototsuji, A.; Balcytis, A.; Linklater, D.; Juodkazis, S.; Tsuboi, Y. Optical Tweezers and Biding at High Irradiation Powers on Black Si. Sci. Rep. 2017, 7, 12298.

(34) Hanasaki, I.; Shoji, T.; Tsuboi, Y. Regular Assembly of Polymer Nanoparticles by Optical Trapping Enhanced with a Random Array of Si Needles for Reconfigurable Photonic Crystals in Liquid. ACS Appl. Nano Mater. 2019, 2, 7637-7643.

(35) Wang, S.-F.; Yuyama, K.; Sugiyama, T.; Masuhara, H. Reflection Microspectroscopic Study of Laser Trapping Assembling of Polystyrene Nanoparticles at Air/Solution Interface. J. Phys. Chem. C 2016, 120 (29), 15578-15585.

(36) Kudo, T.; Yang, S.-J.; Masuhara, H. A Single Large Assembly with Dynamically Fluctuating Swarms of Gold Nanoparticles Formed by Trapping Laser. Nano Lett. 2018, 18 (9), 5846-5853.

(37) Huang, C.-H.; Kudo, T.; Bresoli-Obach, R.; Hofkens, J.; Sugiyama, T.; Masuhara, H. Surface Plasmon Resonance Effect on Laser Trapping and Swarming of Gold Nanoparticles at Interface. Optics Express, 2020, 28, 27727-27735.

(38) Aibara, I.; Hunag, C.-H.; Kudo, T.; Bresoli-Obach, R.; Hofkens, J.; Furbe, A.; Masuhara, H. Dynamic Coupling of Optically Evolved Assembling and Swarming of Gold Nanoparticles with Photothermal Local Phase Separation of Polymer Solution. J. Phys. Chem. C 2020, 124, 16604-16615.

(39) Yuyama, K.; Sugiyama, T.; Masuhara, H. Laser Trapping and Crystallization Dynamics of L-Phenylalanine at Solution Surface. J. Phys. Chem. Lett. 2013, 4 (15), 2436-2440.

(40) Cheng, A.-C.; Masuhara, H.; Sugiyama, T. Evolving Crystal Morphology of Potassium Chloride Controlled by Optical Trapping. J. Phys. Chem. C. 2020, 124, 6913-6921.

(41) Louis, B.; Camacho, R.; Bresoli-Obach, R.; Abakumov, S.; Vandaele, J.; Kudo, T.; Masuhara, H.; Scheblykin, I. G.; Hofkens, J.; Rocha, S. Fast Tracking of Single Emitters in Large Volumes with Nanometer Precision, Optics Express, 2020, 28, 28656-28671.

(42) Ito, S.; Sugiyama, T.; Toitani, N.; Katayama, G.; Miyasaka, H. Application of Fluorescence Correlation Spectroscopy to the Measurement of Local Temperature in Solution under Optical Trapping Condition, J. Phys. Chem. B 2007, 111, 2365-2371.

(43) Misawa, H.; Koshioka, M.; Sasaki, K.; Kitamura, N.; Masuhara, H. Spatial Pattern formation, Size Selection, and Directional Flow of Polymer Latex Particles by Laser Trapping Technique. Chem. Lett., 1991, 469-472.

(44) Nam, H. J.; Jung, D.-Y.; Yi, G.-R.; Choi, H. Close-Packed Hemispherical Microlens Array from Two-Dimensional Ordered Polymeric Microspheres. Langmuir, 2006, 22, 7358-7363.

(45) Shoji, T.; Shibata, M.; Kitamura, N.; Nagasawa, F.; Takase, M.; Murakoshi, K.; Nobuhiro, A.; Mizumoto, Y.; Ishihara, H.; Tsuboi, Y. Reversible Photoinduced 
Formation and Manipulation of a Two-Dimensional Closely Packed Assembly of Polystyrene Nanospheres on a Metallic Nanostructure, J. Phys. Chem. C 2013, 117, 2500-2506

(46) Uwada, T.; Sugiyama, T.; Masuhara, H. Wide-field Rayleigh Scattering Imaging and Spectroscopy of Gold Nanoparticles in Heavy Water under Laser Trapping. $J$.

Photochem. Photobiol. A 2011, 221, 187-193.

(47) Hosokawa, C.; Tsuji, T.; Kishimoto, T.; Okubo, T.; Kudoh, S. N.; Kawano, S. Convection Dynamics Forced by Optical Trapping with a Focused Laser Beam, J. Phys. Chem. C 2020, 124, 8323-8333.

(48) Flores-Flores, F.; Torres-Hurtado, S. A.; Paez, R.; Ruiz, U.; Beltran-Perez, G.; Neale, S. L.; Ramirez-San-Juan, J. C.; Ramos-Garcia, R. Trapping and Manipulation of Microparticles Using Laser-induced Convection Current and Photophoresis. Biomed. Opt. Express 2015, 6 (19), 514-523.

(49) Usman, A.; Chiang, W.Y.; Masuhara, H. Optical trapping and polarization-controlled scattering of dielectric spherical nanoparticles by femtosecond laser pulses. 2012. $J$. Photochem. Photobio. A 234, 83-90.

(50) Chiang, W.Y.; Usman, A.; Masuhara, H. Femtosecond pulse-width dependent trapping and directional ejection dynamics of dielectric nanoparticles, J. Phys. Chem. C 2013. $117,19182-19188$.

(51) Muramatsu, M.; Shen, T.F.; Chiang, W.Y.; Usman, A.; Masuhara, H. Picosecond Motional Relaxation of Nanoparticles in Femtosecond Laser Trapping. J. Phys. Chem. C 2016. 120, 5251-5256.

(52) Chiang, W.Y.; Chen, J. J. K., Usman, A.; Kudo, T.; Xia, K.; Su, J.; Sugiyama, T.; Hofkens, J.; Masuhara, H. 2019. Formation mechanism and fluorescence characterization of a transient assembly of nanoparticles generated by femtosecond laser trapping, J. Phys. Chem. C 123, 27823-27833.

(53) Mondal, D.; Dinda, S.;Bandyopadhyay, S. N.; Goswami, D. Polarization Induced Control of Optical Trap Potential in Binary Liquids. Sci. Report, 2019, 9, 700.

(54) Roy, D.; Mondal, D.; Goswami, D. Structure and Dynamics of Optically Directed SelfAssembly of Nanoparticles. Sci. Report, 2016, 6, 23318.

(55) Mondal, D.; Goswami, D. Controlling and Tracking of Colloidal Nanostructues through Two-Poton Fluorescence. Methods Appl. Fluorescence 2016, 4, 044004.

(56) Mondal, D.; Bandyopadhyay, S. N.; Goswami, D. Elucidating Optical Filed Directed Hierarchical Self-Assembly of Homogeneous versus Heterogeneous Nanoclusters with Femtosecond Optical Tweezers. Plos One 2019, 14, e0223688.

(57) Delgado-Buscalioni, R.; Meléndez, M.; Luis-Hita, J.; Marqués, M. I. ; Sáenz, J. J. Emergence of collective dynamics of gold nanoparticles in an optical vortex lattice. Phys. Rev. E. 2018, 98, 062614-1-062614-8.

(58) Olmos-Trigo, J.; Meléndez, M.; Delgado-Buscalioni, R.; Sáenz, J. J. Sectoral multipole focused beams. Opt. Express 2019, 27 (11), 16384-16394.

(59) Zhang, J.; Guo, T.; Mou, F.; Guau, T. Light-controlled Swarming and Assembly of Colloidal Particles. Micromachines 2018, 9, 88.

(60) Tsai, C.-H. D.; Phan, M. H.; Mizoue, K.; Kaneko, M. Unexpected Beads Alignment in a Microfuidic Channel. Proc. of 2016 IEEE/RSJ International Conference on Intelligent Robots and Systems (IROS) 2016, 5143-5148. 
TOC Graphic

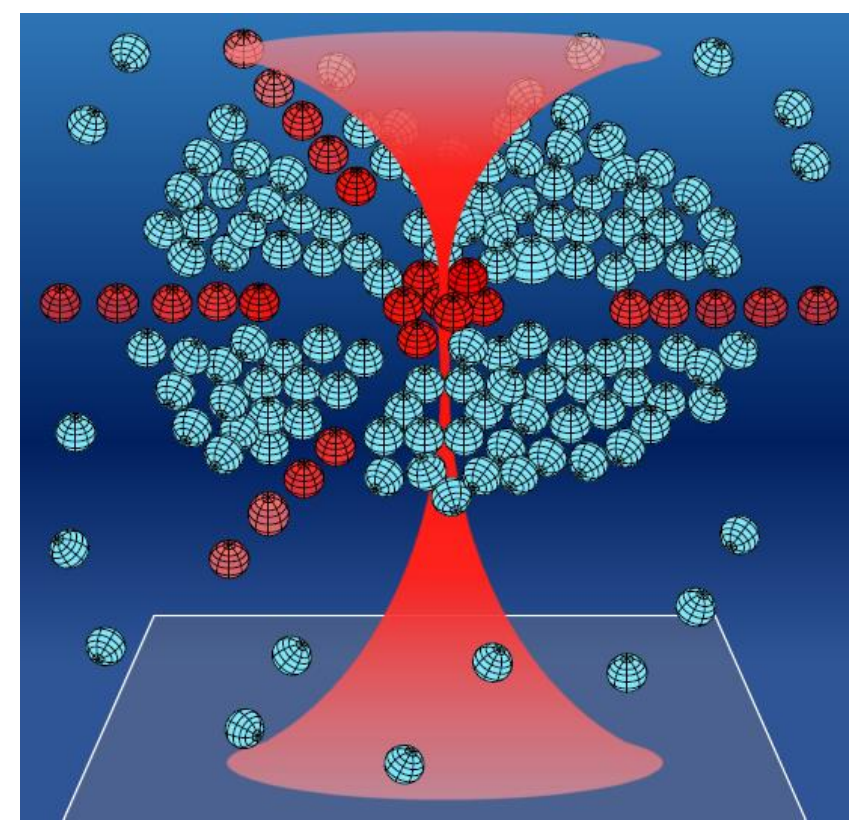




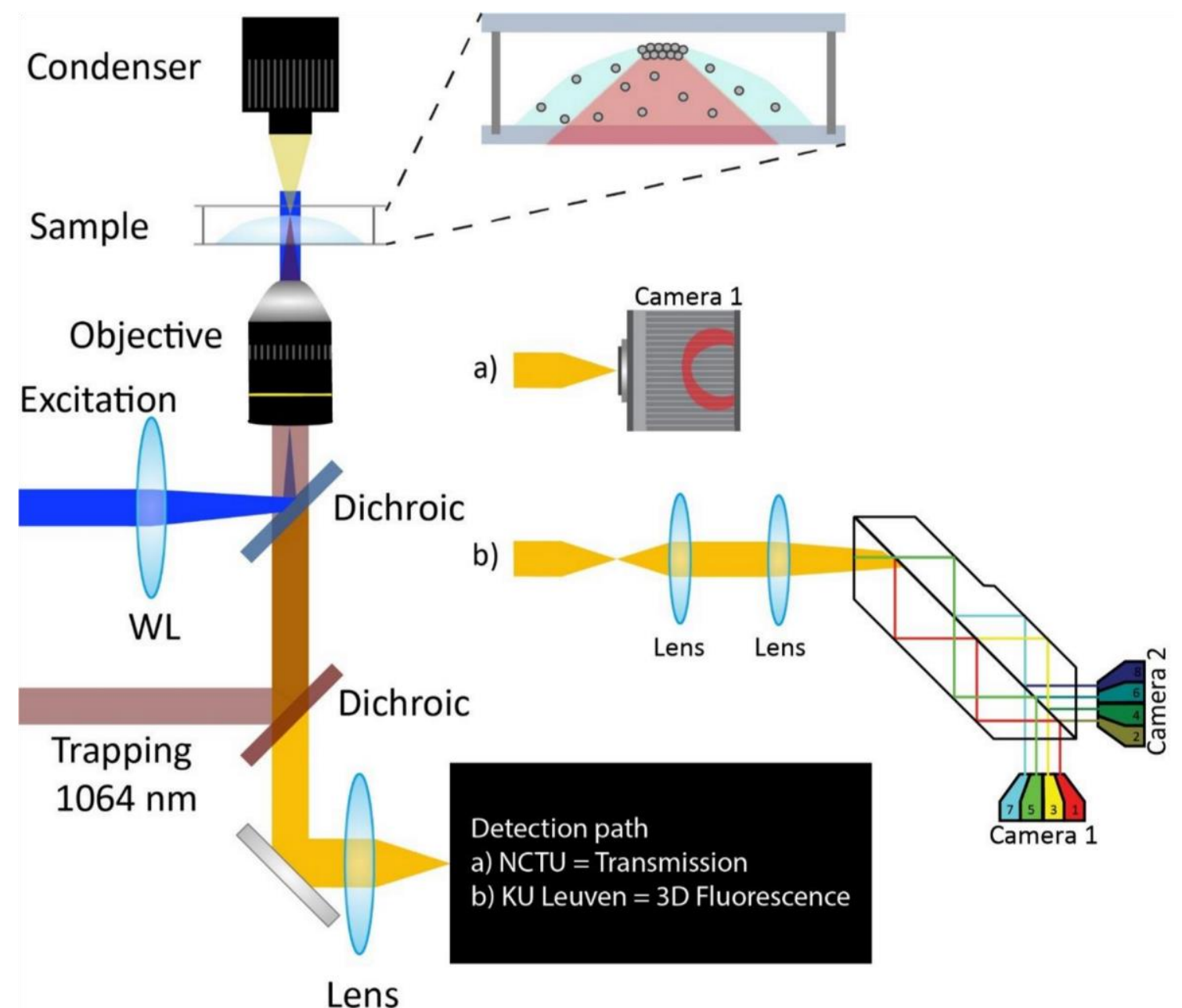

Figure 1. The illustration of the experimental setup for the optical trapping of PS MPs where the two different imaging methods are depicted (See the Experimental). a) Conventional twodimensional transmission or backscattering image is acquired using a CCD camera. b) Multiplane images are acquired after cropping the image with a field stop; splitting the entering image to eight different focus depth images using a proprietary prism and acquired simultaneously using two sCMOS cameras. The dashed line inset shows the illustration of sample a substrate containing the PS MPs, in which the trapping laser is focused at the solution surface. 

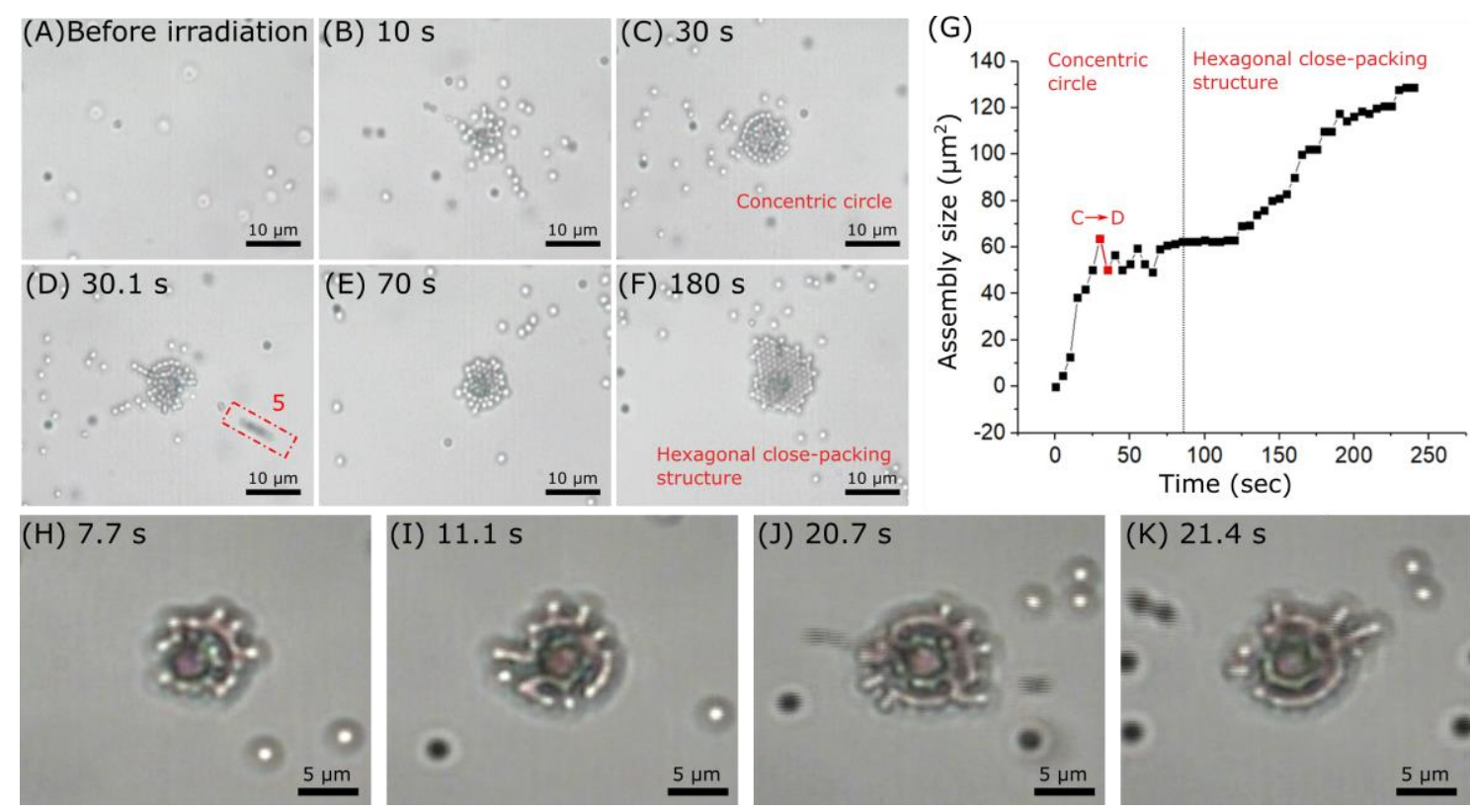

Figure 2. Transmission images of laser trapping-induced formation of a single assembly of 1 $\mu \mathrm{m}$ PS MPs at the solution surface. (A) Before irradiation. (B)-(F) at 10, 30, 30.1, 70, and 180 $\mathrm{s}$ after switching on the $1064 \mathrm{~nm}$ laser, respectively, and one pistol-like ejection with five ejected PS MPs are marked by a red square in (D). (G) The lateral area of the assembly estimated from the corresponding images is plotted against the irradiation time. The inserted C->D corresponds to the sequential images from $(C)$ to $(D) .(H)-(K)$ show the enlarged and detailed structures of the initial assembly (different experiment under the same condition). Laser power is $1.4 \mathrm{~W}$, which corresponds to the power density of about $140 \mathrm{MW} / \mathrm{cm}^{2}$. 

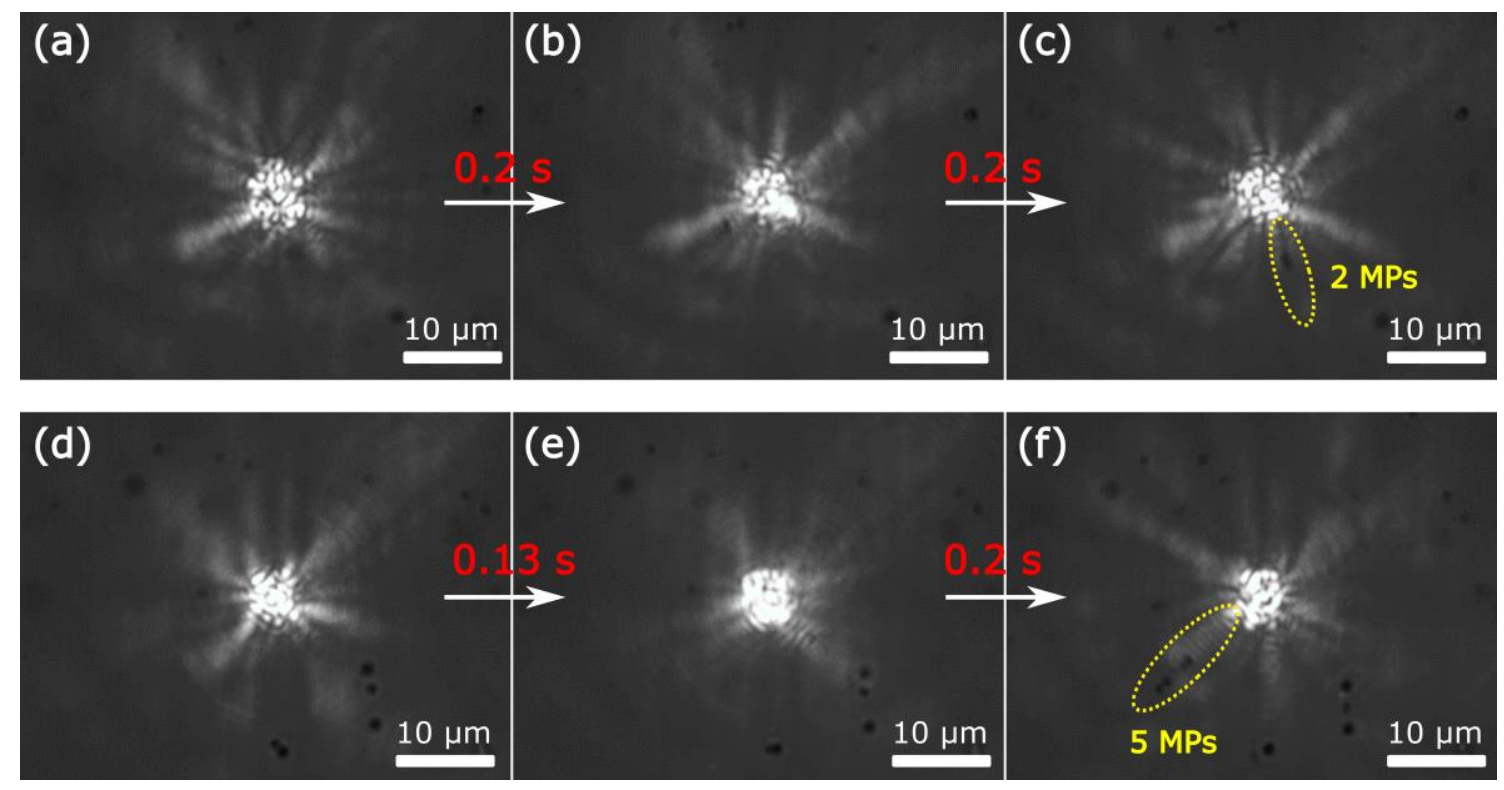

Figure 3. Overlaid backscattering of the $1064 \mathrm{~nm}$ laser and transmission images showing the pistol-like ejection of $1 \mu \mathrm{m}$ PS MPs from the single assembly. In the scattering light, the $1 \mu \mathrm{m}$ PS MPs are identified as black dots. Examining the corresponding images, two and five PS MPs ejected together in a linearly aligned manner are surrounded in a yellow ellipse and the total number of the ejected MPs is written in yellow colour. Laser power is $1.4 \mathrm{~W}$. 

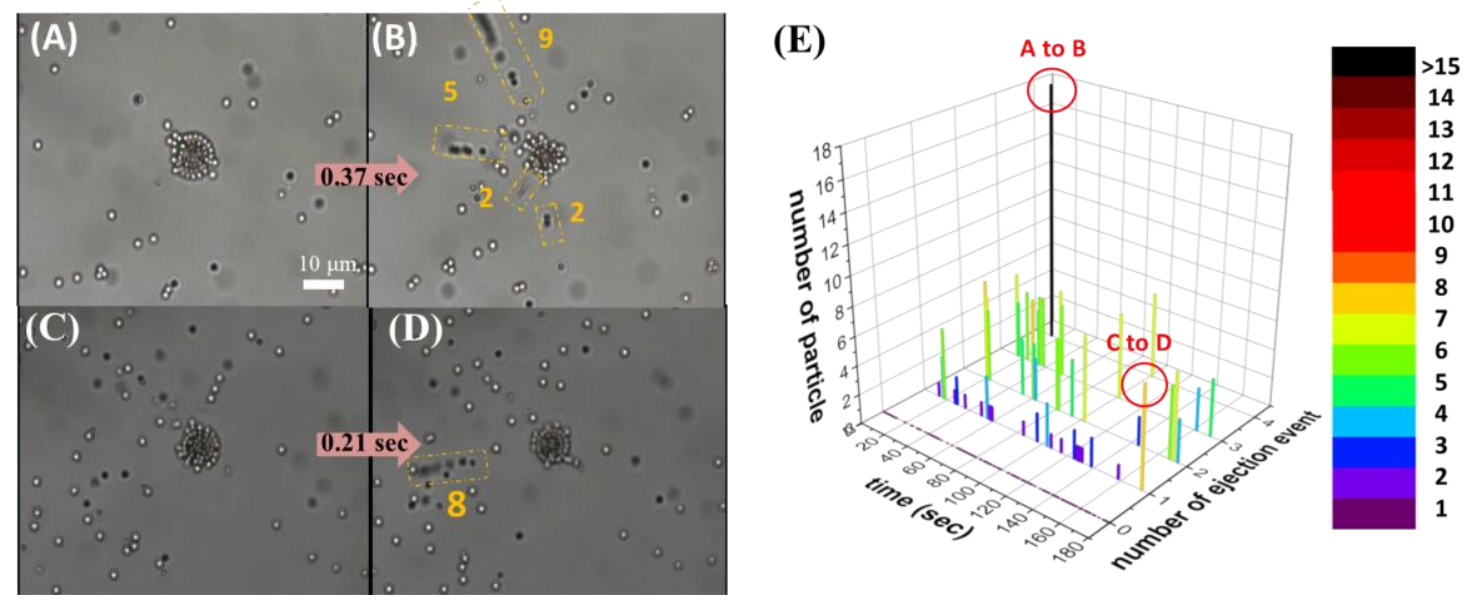

Figure 4. (A)-(D) The MPs ejected together in a linearly aligned manner are surrounded in a yellow-orange coloured square and the number of ejected MPs per ejection event is written in yellow-orange colour. (E) The number of the PS MPs per ejection is plotted as functions of irradiation time and the number of the ejection event in one frame. The colour of the bars in the graph represents the number of PS MPs per each ejection event as given in the right side. Laser power is $1.4 \mathrm{~W}$. 

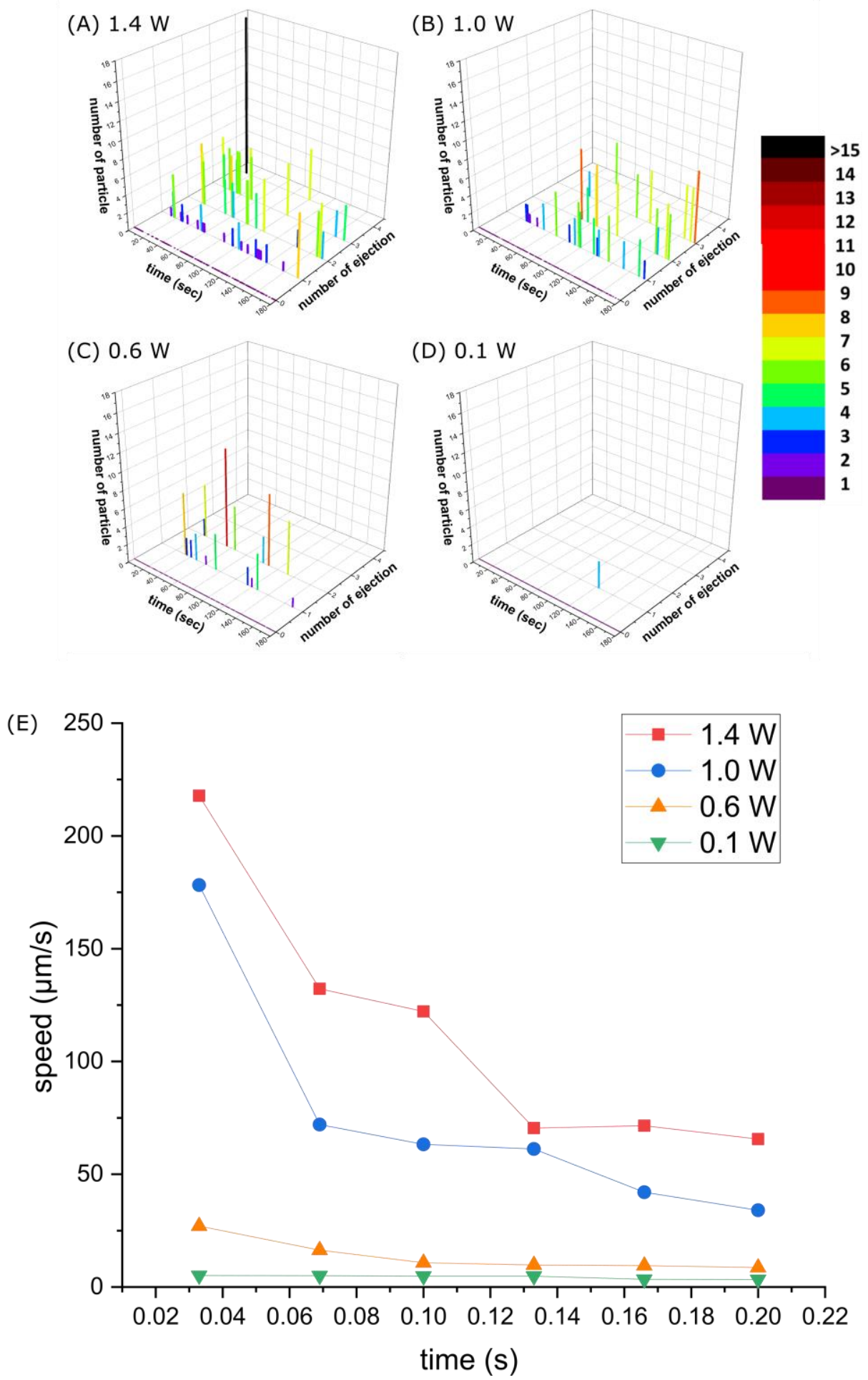

Figure 5. (A)-(D) The number of the PS MPs per ejection is plotted as functions of irradiation time and the number of the ejection event for each recorded frame, which is summarized given for different laser power. (E) The ejected speed of the PS MPs calculated for different laser power (See the text). 


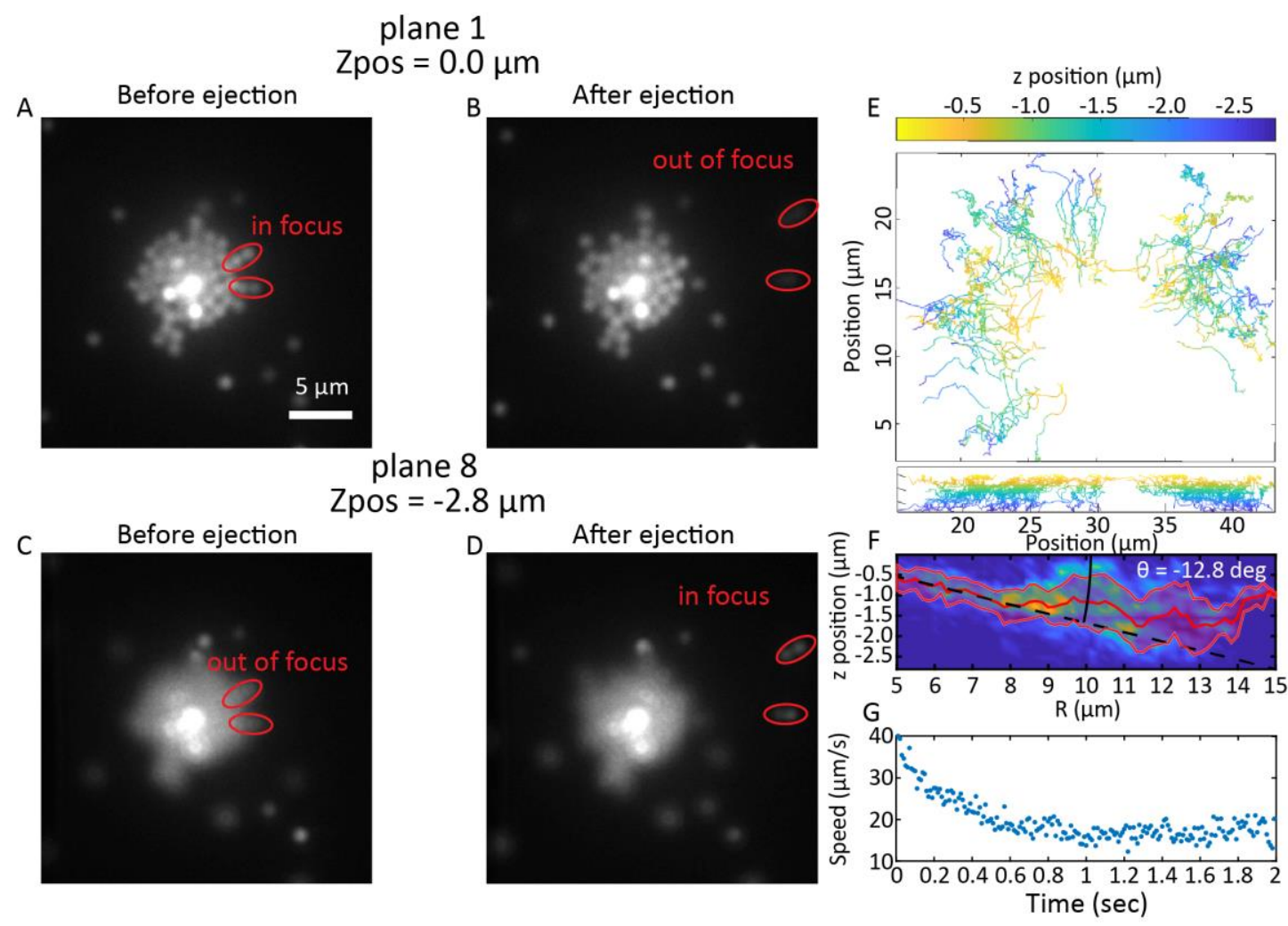

Figure 6. 3D motion analysis of the pistol-like ejection using a fluorescent dye-doped MP. Laser power is $0.36 \mathrm{~W}$. (A)-(D) Representative images at two different depth planes of ( $\mathrm{z}=0.0$ $\mu \mathrm{m}$ (top) and $-2.8 \mu \mathrm{m}$ (bottom)) before and after the pistol-like ejection. (E) 3D tracking traces of the detected pistol-like ejections for $\mathrm{x}-\mathrm{y}$ and $\mathrm{x}-\mathrm{z}$ projections. (F) Probability density function of the pistol-like ejection in the z-direction vs the distance from the focus. The mean trajectory is displayed as a red line and the standard deviation is displayed as a red shadow. (G) The 3D ejection speed of PS MPs. 


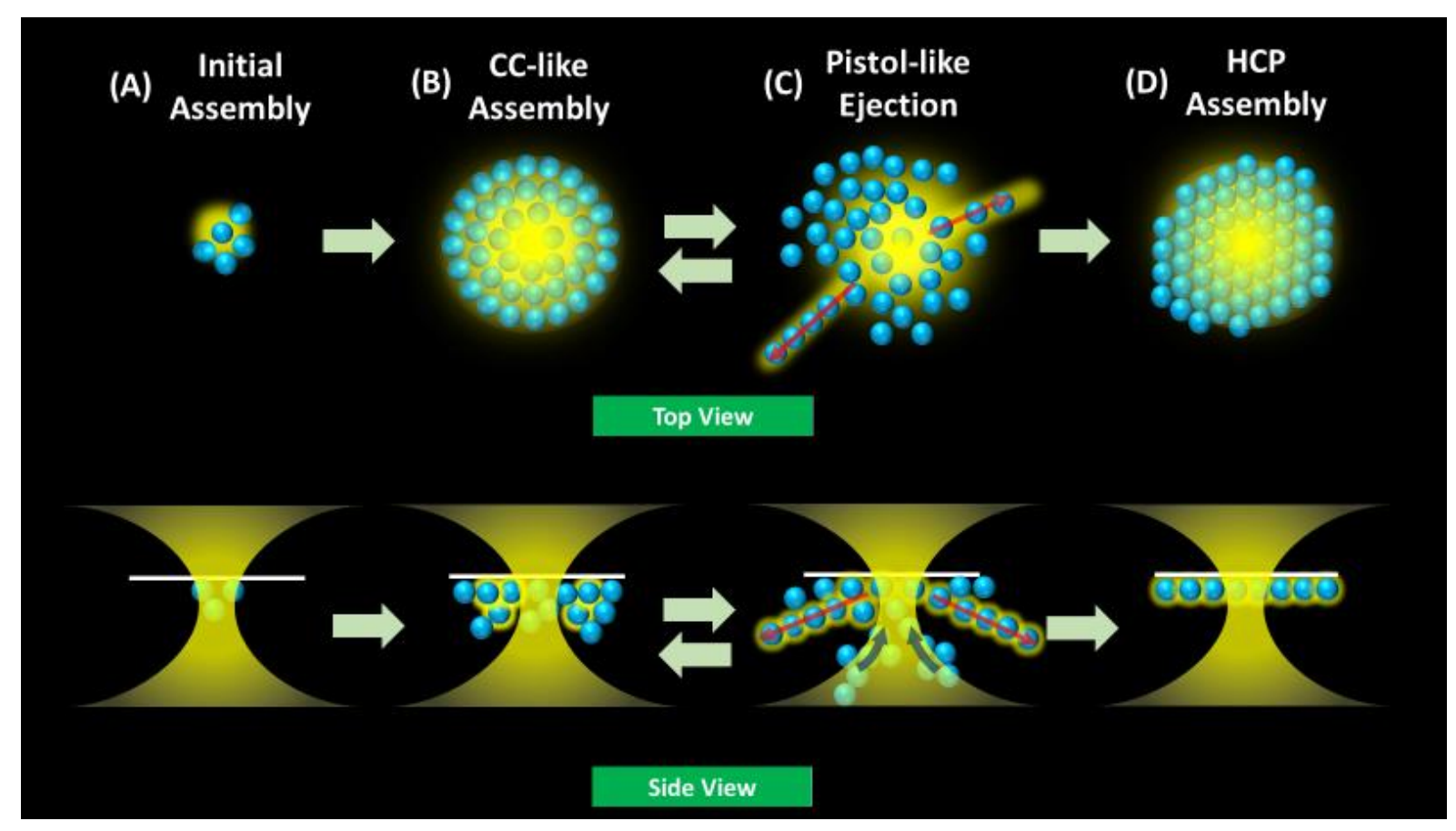

Figure 7. Illustration of dynamics and mechanism of optical force-induced assembling rearrangement, and three-dimensional pistol-like ejection of microparticles at solution surface. Black and red arrows in (C) represent the attracted movement of MPs toward the focus and the pistol-like ejection of MPs in linearly aligned manners, respectively. 